\title{
On Performing Simultaneous Electron Transfer Dissociation and Collision-Induced Dissociation on Multiply Protonated Peptides in a Linear Ion Trap
}

\author{
J. Larry Campbell, James W. Hager, and J. C. Yves Le Blanc \\ MDS Analytical Technologies, Concord, Ontario, Canada
}

We propose a tandem mass spectrometry method that combines electron-transfer dissociation (ETD) with simultaneous collision-induced dissociation (CID), termed ETD/CID. This technique can provide more complete sequence coverage of peptide ions, especially those at lower charge states. A selected precursor ion is isolated and subjected to ETD. At the same time, a residual precursor ion is subjected to activation via CID. The specific residual precursor ion selected for activation will depend upon the charge state and $\mathrm{m} / \mathrm{z}$ of the ETD precursor ion. Residual precursor ions, which include unreacted precursor ions and charge-reduced precursor ions (either by electron-transfer or proton transfer), are often abundant remainders in ETD-only reactions. Preliminary results demonstrate that during an ETD/CID experiment, b, $\mathrm{y}, \mathrm{c}$, and z-type ions can be produced in a single experiment and displayed in a single mass spectrum. While some peptides, especially doubly protonated ones, do not fragment well by ETD, ETD/CID alleviates this problem by acting in at least one of three ways: (1) the number of ETD fragment ions are enhanced by CID of residual precursor ions, (2) both ETD and CID-derived fragments are produced, or (3) predominantly CID-derived fragments are produced with little or no improvement in ETD-derived fragment ions. Two interesting scenarios are presented that display the flexibility of the ETD/CID method. For example, smaller peptides that show little response to ETD are fragmented preferentially by CID during the ETD/CID experiment. Conversely, larger peptides with higher charge states are fragmented primarily via ETD. Hence, ETD/CID appears to rely upon the fundamental reactivity of the analyte cations to provide the best fragmentation without implementing any additional logic or MS/MS experiments. In addition to the ETD/CID experiments, we describe a novel dual source interface for providing front-end ETD capabilities on a linear ion trap mass spectrometer. (J Am Soc Mass Spectrom 2009, 20, 1672-1683) (c) 2009 American Society for Mass Spectrometry

$\mathrm{E}$ lectron-capture dissociation (ECD) [1] and electrontransfer dissociation (ETD) [2] are known for their ability to provide biomolecule sequencing information that is complementary to traditional collisioninduced dissociation (CID) based methods. The ability of both dissociation methods, ECD and ETD, to sequence peptides, while retaining very labile posttranslational modifications (PTMs), is extremely useful. However, while the benefits of these techniques are well documented, there are also some drawbacks.

Fragmentation of doubly protonated peptides via ETD is generally a low efficiency process, due in part to the relatively low charge states present in the reactants of this ion/ion reaction $[3,4]$. It is well known that the higher the total number of charges (i.e., the greater the protonation state), the faster and more exothermic the

Address reprint requests to Dr. J. L. Campbell, MDS Analytical Technologies, 41 Four Valley Drive, Concord, Ontario L4K 4V8, Canada. E-mail: larry.campbell@sciex.com
ETD reaction will be $[3,5]$. Hence, with precursor ions of low charge state, ETD reactions leave behind residual precursor ions, including unreacted precursor ions and charge-reduced precursor ions (either by electron transfer or proton transfer). Several strategies have been developed for sample preparation to circumvent these shortcomings. For example, enzymes other than trypsin have been employed (Asp-N [6]; Lys-C [7, 8]), which generate larger peptides than trypsin, thus leading to the formation of peptide ions of larger $\mathrm{m} / \mathrm{z}$ and charge state $(\leq 5)$. Other strategies have been examined to increase the charge states of peptide ions, including chemical derivatization to add trimethylammonium butyrate (TMAB) groups [9]. However, despite the potential for sample losses or inefficient modification reactions, the presence of fixed-charge sites can actually decrease the efficiency of the ETD reaction.

To address the problem of decreased ETD efficiency for doubly protonated peptide ions, the use of collisional activation in ETD experiments has been explored. 
Supplemental activation of charge-reduced ETD product ions in either a general $[4,10,11]$ or selective [12-14] manner has been used to enhance the formation and detection of ETD-derived fragment ions. This is especially useful when the precursor ions are only doubly charged, as these peptide cations are known for producing few c- and z-type fragment ions in ETD MS/MS.

Another detrimental facet of many ETD reactions is that a large number of precursor ions do not dissociate, including unreacted precursor ions, precursor ions that underwent electron-transfer without dissociation (ET no D), $[3,15]$, or precursor ions that were deprotonated by the ETD reagent anions (PTR products) [16]. ETD reactions are thermodynamically divided into two major reaction channels: electron-transfer and proton transfer. As such, a portion of the total ion/ion reaction will involve proton transfer from the multiply protonated analyte molecule to the anionic reagent $[15,16]$.

In this work, we propose a MS/MS technique, simultaneous ETD/CID, which strives to alleviate the aforementioned disadvantages. This method employs CID performed on selected residual precursor ions of ETD reactions to collect b-, y-, c-, and z-type fragment ions in a single experiment and mass spectrum. The collection of both ETD and CID data in separate experiments has been demonstrated to be advantageous for obtaining higher quality peptide identifications [17-19]. However, it is often unnecessary to collect these data in two distinct mass spectra when (1) there are sufficient precursor ions available to perform both dissociation methods, and (2) there is little or no overlap among all potential fragment ion types for a given dissociated peptide so identification of these ions can be done with reasonable confidence $[20,21]$.

There are many examples of coupling ETD (or ECD) and a thermal activation method (such as CID or IRMPD). Within these experiments, the entire ion population is generally subjected to thermal excitation either before [22], during [4, 23-26], or after [12, 13, 24] the ETD or ECD event. This can result in the suppression of c-and z-type fragment ions via uncontrolled secondary fragmentation by IRMPD. Alternatively, an isolation step can be implemented before subjecting certain ECD product ions to thermal excitation [11,27], but this can extend the cycle time of such an experiment.

The ETD/CID method described herein differs from these combinations of electronic and thermal excitation. The ETD/CID technique employs selective excitation of residual precursor ions by means of application of an auxiliary waveform to the linear ion trap (LIT) in which the ions are trapped during the ETD reaction. Liu and coworkers [26] performed similar experiments on intact multiply protonated insulin proteins. This produces band y-type fragment ions and, in most cases, augments levels of c- and z-type ions. Since deprotonated precursor ions generated by PTR are also available targets, they can be activated as well.

We have also explored the generation and introduction of intense reagent anion populations by using a unique source for front-end ETD (FE-ETD). Bright sources of reagent anions are key to converting analyte cations into useful diagnostic product ions by ETD (as well as any ion/ion reactions) [6, 28]. ETD reagent anions produced in vacuo $[6,29]$ meet this criterion, but there are limitations to this design. For example, there are unwanted costs of external ion optics and differential pumping, as well as the inherent limited flexibility of these ion sources. While an ETD reagent ion can be generated, the production of a different reagent anion requires disassembly and, in some cases, venting of the mass spectrometer to atmosphere.

One solution to overcome this problem has been the generation of analyte and reagent anions at atmospheric pressure, and sequential introduction these ions into the mass spectrometer through a common orifice. While a FE-ETD configuration can have greater flexibility to change between different reagent anions, most examples of FE-ETD have exhibited long cycle times in the range of 3-6 s [30-32] due primarily to difficulty in acquiring sufficient populations of ETD reagent anions. For example, the dual source of Williams and coworkers [31] employs two ESI emitters; one is used to generate positive analyte ions (peptides), while the other is used to generate the precursor of an ETD reagent anion. The precursor ion, 9-anthracenecarboxylate anion $(\mathrm{m} / \mathrm{z} 221)$, is then subjected to CID to form the actual ETD reagent anion: 9-dehydroanthracene anion $(\mathrm{m} / \mathrm{z}$ 177). Unfortunately, despite the fast switching abilities of this source, this FE-ETD configuration was reported [30] to experience poor anion electron-transfer efficiency, more PT than ET reactions (a ratio of $\sim 7: 1$ ), and long acquisition times $(\sim 6 \mathrm{~s})$.

To avoid these negative effects, we employed a modular, multipurpose ion source for our FE-ETD experiments based upon a DuoSpray ion source, which can generate analyte ions either by ESI or APCI [33]. However, we have altered the APCI source to operate independently in negative mode as a source of reagent anions (e.g., azobenzene radical anions). The voltages and nebulizing gases of these two probes can be pulsed as desired.

\section{Experimental}

\section{Materials}

Methanol was purchased from Mallinckrodt Baker (Phillipsburg, NJ, USA). All other chemicals were obtained from Sigma-Aldrich (St. Louis, MO, USA) and were used as obtained. The only exceptions were the peptide KGAILKGAILR that was obtained from SynPep (Dublin, CA. USA) and the heptapeptides (WVYIHPK, HVYIHPF, and RVGIHPF) obtained from Professor Ivan Chu (University of Hong Kong). Peptides were dissolved to $1 \mu \mathrm{M}$ in a $50 / 50$ methanol/water, $0.1 \%$ formic acid solution for positive ESI, while the working solution of azobenzene was prepared in neat acetonitrile to a concentration of $1-5 \mathrm{mg} / \mathrm{mL}$. 
(a)

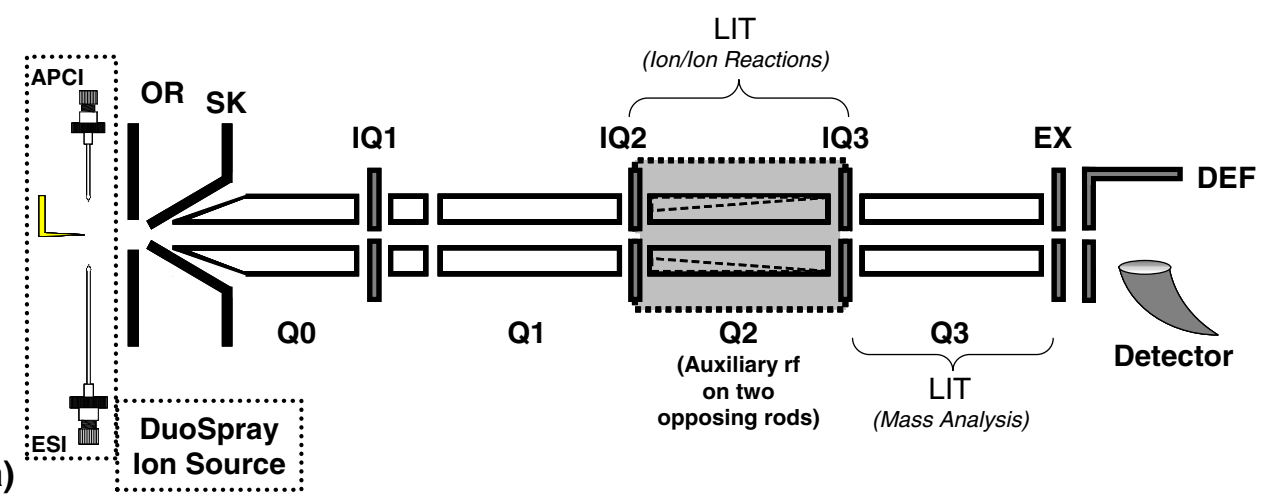

(b)

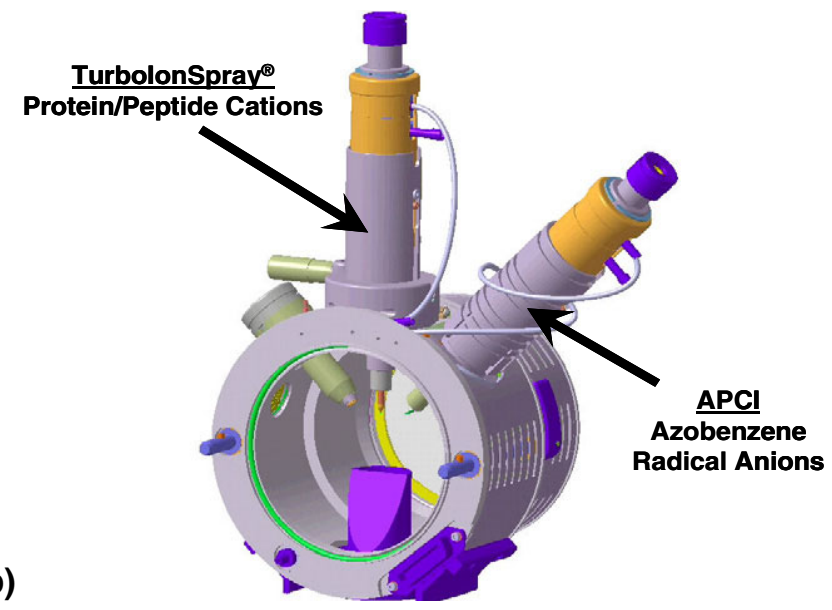

Figure 1. (a) Schematic of the FE-ETD instrument, including the DuoSpray ion source, used in the ETD/CID experiments. (b) Schematic of the DuoSpray ion source.

\section{Mass Spectrometry}

All experiments were performed using a prototype version of a 4000 QTRAP mass spectrometer [34] (Applied Biosystems/MDS Analytical Technologies, Concord, ON, Canada) (Figure 1a) that has been modified to generate and store both positive and negative ions for ion/ion reactions. The ETD experiments were performed in a front-end (FE-ETD) configuration [31, 32, 35] in which both reagent anions (such as azobenzene radical anions) as well as peptide cations were introduced into the mass spectrometer through a common orifice.

The Q0 and Q1 quadrupole arrays are capacitively coupled, and are operated at a drive frequency of $816 \mathrm{kHz}$.
The Q2 section of the mass spectrometer consists of a LINAC collision cell with auxiliary electrodes that provide an axial potential $[36,37]$. The LINAC function was turned off for the duration of the experiment, except during the injection of reagent anions and for the ejection of cations from Q2 after ETD/CID reactions. A base pressure of $\sim 3$ mTorr of nitrogen was established within Q2, which aided in cooling both reagent and analyte ions before and during ETD and ETD/CID reactions. Both the Q2 and Q3 quadrupoles, which are capacitively coupled, are configured as LITs and operated at a drive frequency of $816 \mathrm{kHz}$. In these experiments, Q2 was used as the ion/ion reaction region and Q3 was employed as the mass analyzer.

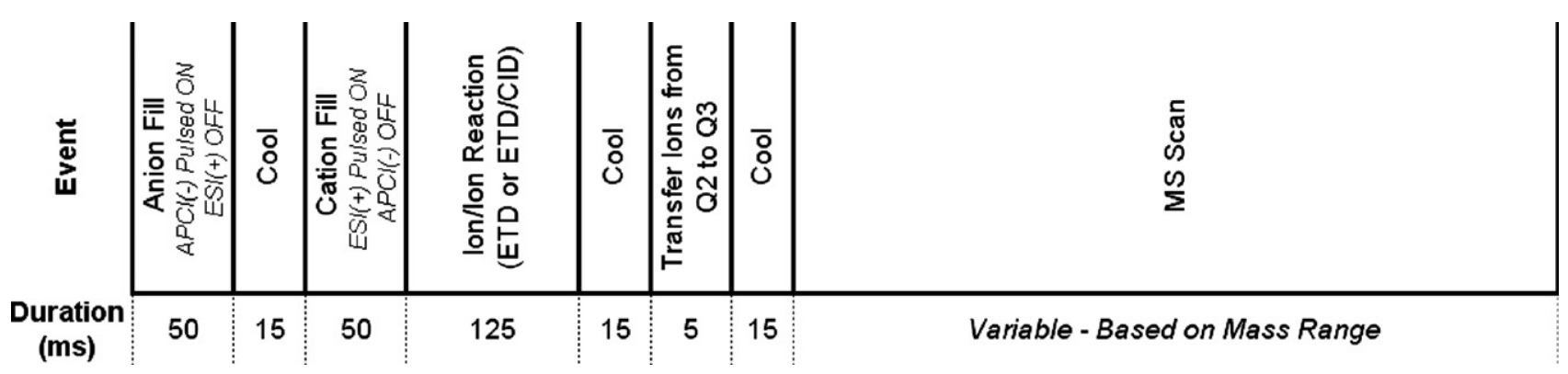

Scheme 1. Timing diagram for a typical ETD (or ETD/CID) experiment, with events and their relevant times indicated. 


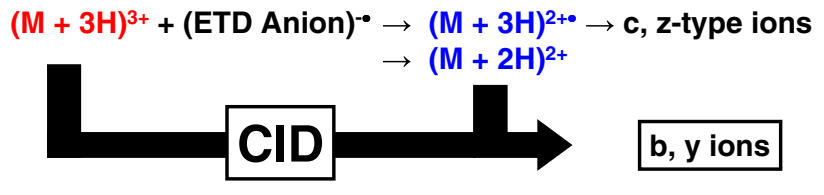

Scheme 2. Flow chart depicting the selection of a triply protonated peptide $\mathrm{M}$ for fragmentation by ETD, as well as the potential target cations for CID. These potential target cations are residual precursor ions remaining after the ETD reaction.

The ionization source is a modified DuoSpray ion source (Figure 1b) that comprises two independent methods of ion generation: an electrospray (ESI) source and an atmospheric pressure chemical ionization (APCI) source. The ESI source was operated in positive ion mode at $+4500 \mathrm{~V}$ and generated cations from the peptide and protein samples. The APCI source, which was operated in negative ion mode at $-3500 \mathrm{~V}$, generated azobenzene radical anions ( $m / z$ 182). Each source is equipped with an independently controlled high voltage power supply (Applied Kilovolts, Worthing, UK). The voltages and the nebulizing gases for both the ESI and APCI sources could be pulsed on during generation of desired ion populations and pulsed off when not needed. The time required for both power supplies to achieve the desired voltages is $\sim 2$ ms. Individual syringe pumps (Harvard Apparatus, Holliston, MA, USA) were used to supply peptide/protein solutions and azobenzene solution at flow rates ranging from 1 to $10 \mu \mathrm{L} / \mathrm{min}$.

All data acquisition and instrument control, excluding the waveform generators, was performed by using
MS Expo 3.11, software that was developed for in-house use. Mass spectra were analyzed and peptides sequenced by using the Analyst/BioAnalyst 1.5 software package (MDS Analytical Technologies/Applied Biosystems, Concord, ON, Canada).

\section{Mutual Storage Mode ETD Experiments}

The timeline of a typical mutual storage mode [35] ETD experiment is displayed in Scheme 1. It begins with the generation and trapping of the ETD reagentazobenzene radical anions. Both the APCI voltage and nebulizing gas are pulsed on for $50 \mathrm{~ms}$, during which time azobenzene radical anions $(m / z 182)$ are generated in the source, passed through Q0, mass resolved by Q1 ( $\sim 4$ Th window), and finally stored in Q2. During a 15-ms cooling step, the APCI voltage, and nebulizing gas are turned off, and the positive ESI voltage and nebulizing gas are pulsed on. For $50 \mathrm{~ms}$, analyte cations (i.e., multiply protonated peptide molecules) are generated in an analogous manner, also mass resolved $(\sim 2$ Th window), and stored with the azobenzene radical anions in Q2.

The simultaneous storage of both positive and negative ions in Q2 was afforded by using auxiliary $\mathrm{rf}$ voltages $\left(200 \mathrm{kHz}, 650 \mathrm{~V}_{p-p}\right)$ applied to two lenses, IQ2 and IQ3, which bookend Q2. These auxiliary voltages, provided by two Agilent 33120A waveform generators (Agilent Technologies, Santa Clara, CA, USA), are maintained for $\sim 125 \mathrm{~ms}$, during which ion/ion chemistry can occur within the Q2 linear ion

Table 1. Data outlining the performance of ETD-only (left-hand side of the table) and ETD/CID (right-hand side) for the candidate peptides/proteins, including \%ETD and sequence coverage, which is listed as the number of c- and z-type ions identified of the total possible number of fragments (ETD-only), as well as the residual precursor ion selected for activation and the sequence coverage (listed as the number of $b_{-}, y_{-}, c_{-}$, and z-type ions identified of the total possible number of fragments) (ETD/CID)

\begin{tabular}{|c|c|c|c|c|c|c|}
\hline Peptide (Protein Name) & $\begin{array}{l}\text { Precursor ion } \\
\qquad(\mathrm{m} / \mathrm{z})\end{array}$ & $\begin{array}{c}\% \\
\text { ETD }\end{array}$ & $\begin{array}{l}\text { ETD-only } \\
\text { sequence } \\
\text { coverage } \\
\text { (obs./theory) }\end{array}$ & $\begin{array}{l}\text { Precursor ion } \\
\qquad(\mathrm{m} / \mathrm{z})\end{array}$ & $\begin{array}{l}\text { Activated ion } \\
\qquad(\mathrm{m} / \mathrm{z})\end{array}$ & $\begin{array}{c}\text { ETD/CID } \\
\text { sequence } \\
\text { coverage } \\
\text { (obs./theory) }\end{array}$ \\
\hline WVYIHPK & $\begin{array}{c}(\mathrm{M}+2 \mathrm{H})^{2+} \\
\mathrm{m} / \mathrm{z} 472\end{array}$ & 22.3 & $6 / 13$ & $\begin{array}{c}(\mathrm{M}+2 \mathrm{H})^{2+} \\
\mathrm{m} / \mathrm{z} 472\end{array}$ & $\begin{array}{c}(\mathrm{M}+2 \mathrm{H})^{+} \\
m / z 472\end{array}$ & $\begin{array}{c}17 / 27 \\
(6-E T D, 11-C I D)\end{array}$ \\
\hline HVYIHPF & $\begin{array}{c}(\mathrm{M}+2 \mathrm{H})^{2+} \\
m / 2457\end{array}$ & 9.6 & $7 / 13$ & $\begin{array}{c}(\mathrm{M}+2 \mathrm{H})^{2+} \\
m / z 457\end{array}$ & $\begin{array}{c}(\mathrm{M}+2 \mathrm{H})^{+} \\
m / z 457\end{array}$ & $\begin{array}{c}18 / 27 \\
(6-E T D, 12-C I D)\end{array}$ \\
\hline RVGIHPF & $\begin{array}{c}(\mathrm{M}+2 \mathrm{H})^{2+} \\
m / z 413\end{array}$ & 31.8 & $8 / 13$ & $\begin{array}{c}(\mathrm{M}+2 \mathrm{H})^{2+} \\
m / z 413\end{array}$ & $\begin{array}{c}(\mathrm{M}+2 \mathrm{H})^{+} \\
\mathrm{m} / \mathrm{z} 413\end{array}$ & $\begin{array}{c}19 / 27 \\
\text { (8-ETD, 11-CID) }\end{array}$ \\
\hline DRVYIHPFHL (Angiotensin I) & $\begin{array}{c}(\mathrm{M}+2 \mathrm{H})^{2+} \\
m / z 649\end{array}$ & 19.6 & $5 / 19$ & $\begin{array}{c}(\mathrm{M}+2 \mathrm{H})^{2+} \\
m / 2649\end{array}$ & $\begin{array}{c}(\mathrm{M}+2 \mathrm{H})^{+} \\
\mathrm{m} / \mathrm{z} 649\end{array}$ & $\begin{array}{c}21 / 39 \\
(6-E T D, 11-C I D)\end{array}$ \\
\hline DRVYIHPF (Angiotensin II) & $\begin{array}{c}(\mathrm{M}+2 \mathrm{H})^{2+} \\
\mathrm{m} / \mathrm{z} 524\end{array}$ & 26.1 & $7 / 15$ & $\begin{array}{c}(\mathrm{M}+2 \mathrm{H})^{2+} \\
\mathrm{m} / \mathrm{z} 524\end{array}$ & $\begin{array}{r}(\mathrm{M}+\mathrm{H})^{+} \\
m / z 524\end{array}$ & $\begin{array}{c}18 / 31 \\
(11-E T D, 7-C I D)\end{array}$ \\
\hline $\begin{array}{l}\text { EGVNDNEEGFFSAR } \\
\left.\text { ([Glu }{ }^{1}\right] \text {-fibrinopeptide B) }\end{array}$ & $\begin{array}{c}(\mathrm{M}+2 \mathrm{H})^{2+} \\
m / z 786\end{array}$ & 5.9 & $3 / 28$ & $\begin{array}{c}(\mathrm{M}+2 \mathrm{H})^{2+} \\
m / z 786\end{array}$ & $\begin{array}{c}(\mathrm{M}+2 \mathrm{H})^{+} \\
\mathrm{m} / \mathrm{z} 786\end{array}$ & $\begin{array}{c}31 / 56 \\
\text { (7-ETD, 24-CID) }\end{array}$ \\
\hline KGAILKGAILR & $\begin{array}{c}(\mathrm{M}+3 \mathrm{H})^{3+} \\
\mathrm{m} / \mathrm{z} 380\end{array}$ & 66.1 & $20 / 22$ & $\begin{array}{c}(\mathrm{M}+3 \mathrm{H})^{3+} \\
\mathrm{m} / \mathrm{z} 380\end{array}$ & $\begin{array}{c}(\mathrm{M}+3 \mathrm{H})^{3+} \\
\mathrm{m} / \mathrm{z} 380\end{array}$ & $\begin{array}{c}39 / 44 \\
(20-E T D, 19-C I D)\end{array}$ \\
\hline RPKPQQFFGLM (Substance P) & $\begin{array}{c}(\mathrm{M}+3 \mathrm{H})^{3+} \\
\mathrm{m} / \mathrm{z} 450\end{array}$ & 60.5 & $17 / 21$ & $\begin{array}{c}(\mathrm{M}+3 \mathrm{H})^{3+} \\
\mathrm{m} / \mathrm{z} 450\end{array}$ & $\begin{array}{c}(\mathrm{M}+3 \mathrm{H})^{2+} \\
\mathrm{m} / \mathrm{z} 450\end{array}$ & $\begin{array}{c}32 / 43 \\
\text { (18-ETD, 14-CID) }\end{array}$ \\
\hline $\begin{array}{l}\text { GIGAVLKVLTTGLPALISWIKRKROQ } \\
\text { (Melittin) }\end{array}$ & $\begin{array}{c}(\mathrm{M}+5 \mathrm{H})^{5+} \\
m / z 570\end{array}$ & 56.8 & $37 / 51$ & $\begin{array}{c}(\mathrm{M}+5 \mathrm{H})^{5+} \\
m / 2570\end{array}$ & $\begin{array}{c}(\mathrm{M}+4 \mathrm{H})^{4+} \\
m / z 712\end{array}$ & $\begin{array}{c}46 / 103 \\
(37-E T D, 9-C I D)\end{array}$ \\
\hline $\begin{array}{l}\text { FQ(pS)EEQQQTEDELQDK } \\
\text { (Monophosphopeptide from } \beta \text {-casein) }\end{array}$ & $\begin{array}{c}(\mathrm{M}+3 \mathrm{H})^{3+} \\
\mathrm{m} / \mathrm{z} 688\end{array}$ & 12.8 & $16 / 32$ & $\begin{array}{c}(\mathrm{M}+3 \mathrm{H})^{3+} \\
\mathrm{m} / \mathrm{z} 688\end{array}$ & $\begin{array}{c}(\mathrm{M}+2 \mathrm{H})^{2+} \\
m / z 1032\end{array}$ & $\begin{array}{c}60 / 64 \\
(30-E T D, 30-C I D)\end{array}$ \\
\hline
\end{tabular}




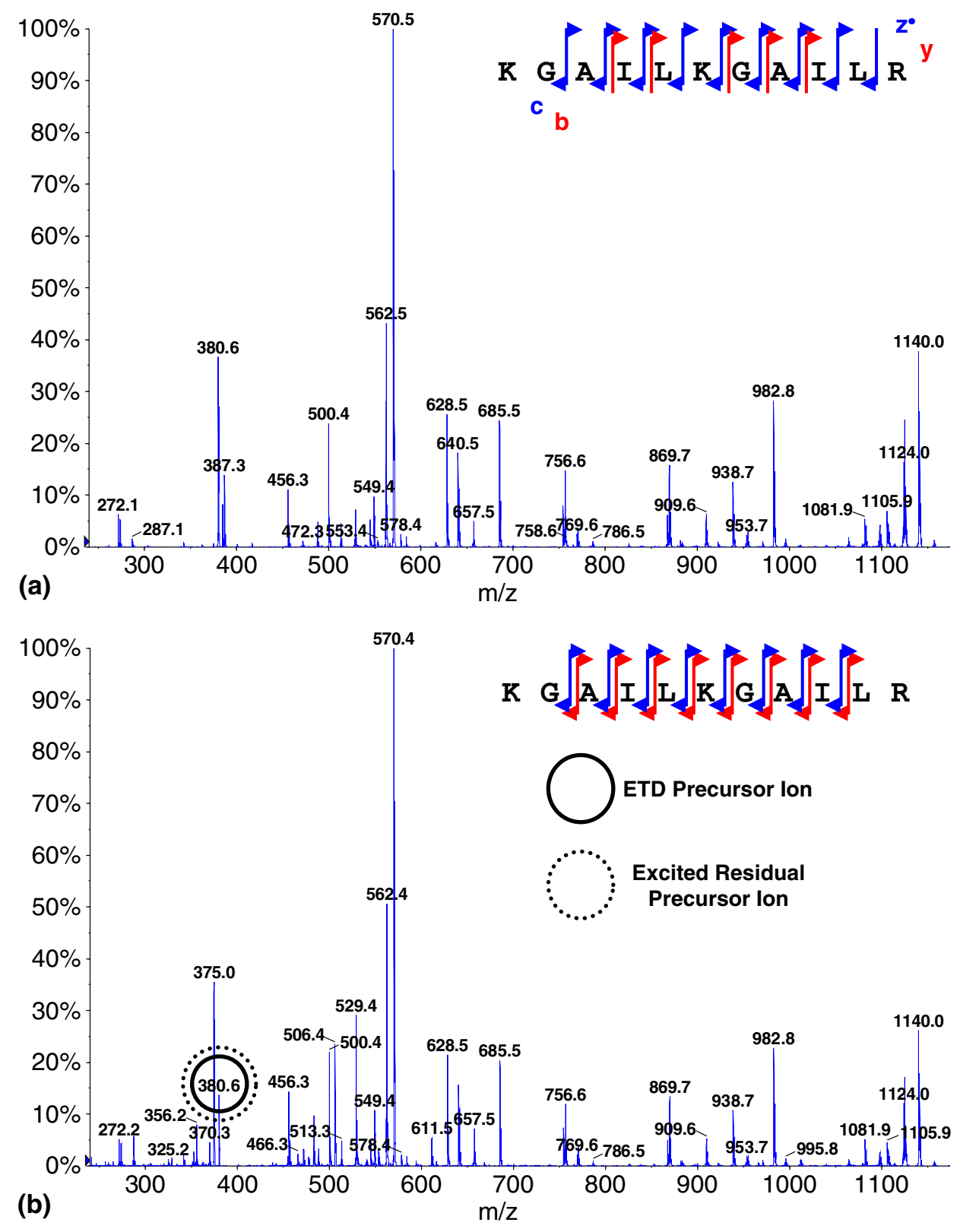

Figure 2. (a) ETD and (b) ETD/CID of the triply protonated peptide of KGAILKGAILR. The ions selected for ETD and CID are indicated by the solid and dotted circles, respectively.

trap. After an additional cooling step of $15 \mathrm{~ms}$, the cations trapped within Q2 are transferred to Q3, trapped and cooled for an additional $15 \mathrm{~ms}$, then scanned out of Q3 by MSAE [38] at a rate of 1000 $\mathrm{Th} / \mathrm{s}$. The cycle time of these experiments was between 0.5 and $1.5 \mathrm{~s}$, which is amenable to LC/MS/MS data acquisition. The mass spectra shown here are averaged over 25-100 individual scans.

\section{Simultaneous ETD/CID}

The experimental setup and timing for simultaneous ETD/CID is quite similar to the aforementioned mutual trapping mode ETD experiment. The princi- ple distinction is the application of auxiliary rf voltage on two opposing rods of the Q2 array during the ETD reaction time. The auxiliary $\mathrm{rf}$ voltage is provided by an Agilent 33220A waveform generator that provides signals of varying frequency and amplitude. The secular frequencies of the ions were calculated using SxStability, a program developed in-house. Alternative strategies that could also be explored include the excitation of two or more secular frequencies during the ETD/CID experiment using a tailored waveform.

The frequency and amplitude of this excitation event was chosen to provide maximum fragmentation of any residual precursor ions (vide supra) during the ETD 

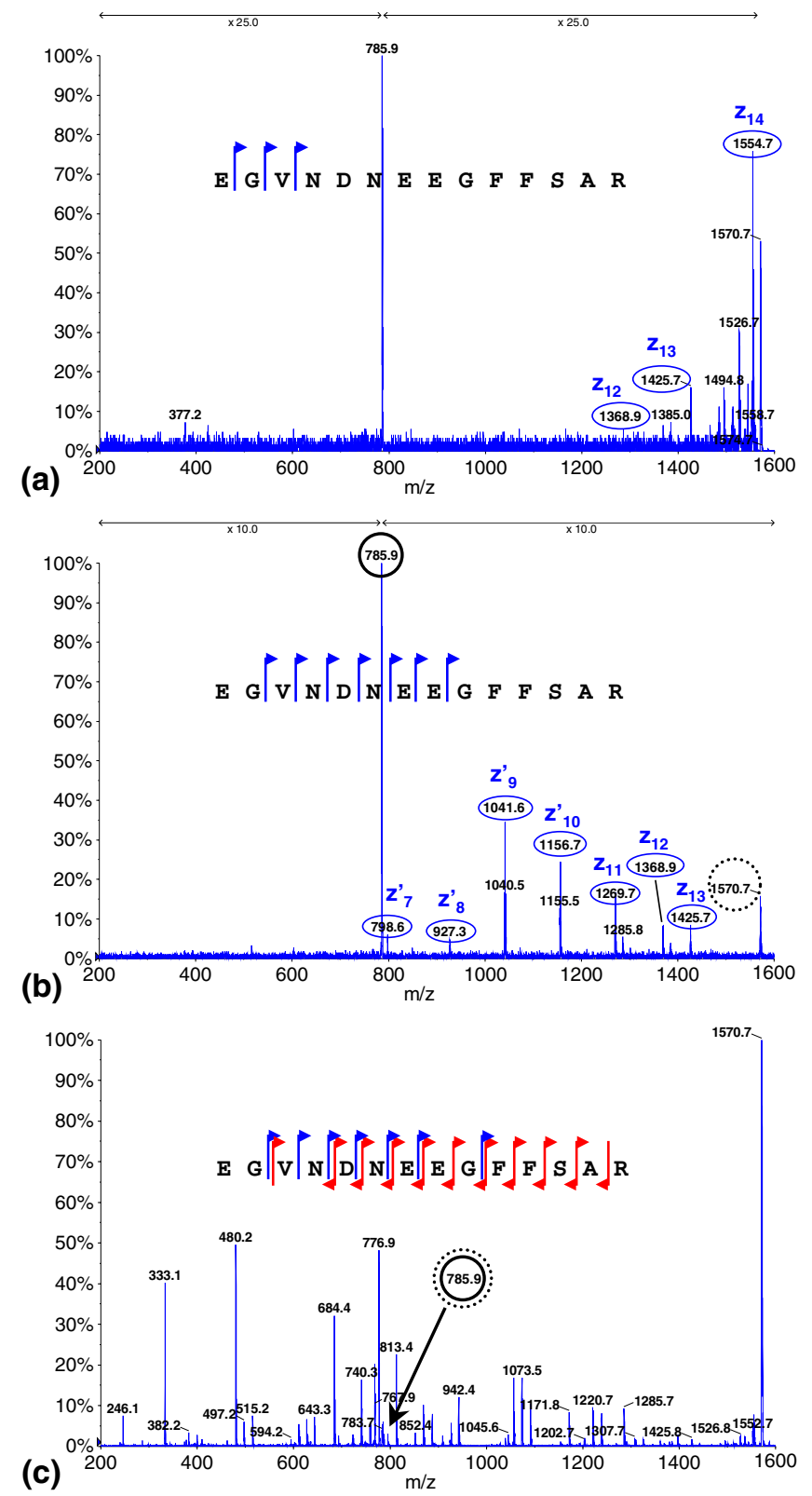

Figure 3. (a) ETD of $(\mathrm{M}+2 \mathrm{H})^{2+}$ ion of $\left[\mathrm{Glu}^{1}\right]$-fibrinopeptide $\mathrm{B}$; $(\mathbf{b})$ ETD of $(\mathrm{M}+2 \mathrm{H})^{2+}$ ion of [Glu1]-fibrinopeptide $\mathrm{B}$ with activation of the charge-reduced precursor ion $(\mathrm{M}+\mathrm{H})^{+}$; $(\mathrm{c})$ ETD of $(\mathrm{M}+2 \mathrm{H})^{2+}$ ion of $\left[\mathrm{Glu}^{1}\right]$-fibrinopeptide $\mathrm{B}$ with activation of the residual precursor ion $(\mathrm{M}+$ $2 \mathrm{H})^{2+}$. The ions selected for ETD and CID are indicated by the solid and dotted circles, respectively.

reaction (Scheme 2). In a separate experiment, CID was performed on the potential target cations and settings were recorded that resulted in the fragmentation of the precursor ion with retention of $\sim 10 \%$ of the initial precursor ion signal. Auxiliary excitation was carried out at low q-values $(\mathrm{q} \sim 0.2-0.3)$.

As stated above, the activation frequency of the targeted analyte cations was set in Q2 when only the positively charged precursor ions were present. However, for the ETD/CID experiment, a large population of reagent anions was trapped in the LIT before the cations were injected. The presence of this large popu- lation of anions is sufficient to shift the realized secular frequency of the cations by several kilohertz (data not shown) [39]. Under these conditions, precursor ions are able to undergo ET reactions with the azobenzene anions until the anion population is sufficiently depleted. At this point, the influence of the electric field induced by the anions is diminished, and the precursor cation's secular frequency again matches the anion-free state. At this point, thermal activation of the precursor cations occurs, and CID fragments are formed and trapped. Further study of this phenomenon is currently underway. 


\section{Results and Discussion}

\section{DuoSpray Ion Source as an FE-ETD Source}

The production of azobenzene is quite stable and intense, despite the pulsed nature of the APCI part of the DuoSpray ion source. Typically, the APCI corona voltage is pulsed to $-3500 \mathrm{~V}$ and nebulizing gas to $50 \mathrm{psi}$. This 50 ms-long event, which marks the beginning of every experiment scan cycle, yields azobenzene reagent anion signal of strong intensity and good stability (data not shown)-a desirable quality for longer analytical ETD experiments.

The overall performance of this FE-ETD instrument has been assessed in several areas. One such measure is the experiment's cycle time. The experiments described herein have cycle times between 0.5 and $1.5 \mathrm{~s}, \sim 2$ to 4 times faster than previously described FE-ETD experiments [30-32]. This improved time efficiency is due to several factors, including the stability and intensity of the anion source and the large reaction volume present in this $22.5 \mathrm{~cm}$-long linear ion trap.

Aside from cycle time, the quality of data from this FE-ETD instrument can be assessed by examining the efficiency of the overall ETD process (\% ETD) $[13,16]$ for some candidate peptide ions; \% ETD is calculated as the sum of all signals from expected ETD products divided by the sum of all signals derived from other ion/ion reactions, including charge-reduced precursor ions:

$$
\% \mathrm{ETD}=\frac{\sum \mathrm{c}, \mathrm{z}^{\bullet} \text { fragments }}{\sum \text { All Other Ion/Ion Reaction Products }}
$$

The \% ETD for these candidate peptide ions, along with the sequences and $\mathrm{m} / \mathrm{z}$ values of the precursor ions, are listed in Table 1. In addition to \% ETD, the sequence coverage afforded by ETD is given for the peptide ions. The theoretical maximum number of possible c- and z-type ions excludes c-type ions that cannot form via cleavage at Xxx-Pro (X-P) bonds. No forms of auxiliary activation were applied to any of the ions during these ETD-only experiments.

These \% ETD results agree with similar ETD-only experiments [4] and reveal limitations of using ETD for sequencing doubly protonated peptides, although ETD of highly protonated peptides is an efficient method. This problem has been well studied [12-14], and now supplemental activation of charge-reduced precursor ions is widely employed. However, even for peptide ions that fragment well by ETD (e.g., KGAILKGAILR, Figure 2a), there remains an abundance of unreacted precursor ions $(\mathrm{m} / \mathrm{z} 380.6)$, as well as charge-reduced precursor ions $(m / z 570.4)$.

\section{Rationale Behind Simultaneous ETD/CID}

Upon examination of the FE-ETD mass spectra, two facts became apparent: (1) there was generally an abundance of residual precursor ion (or charge-reduced precursor ions) remaining at the end of ETD-only reactions, and (2) there was not much overlap between the $\mathrm{m} / \mathrm{z}$ values of the ETD fragment ions and the theoretical CID-generated fragment ions for these peptides. These two facts meant that both fragmentation modes could conceivably be combined into a single process, ETD/CID, which improves experimental cycle time with the elimination of an added step of mass analysis.

\section{ETD/CID of Doubly and Triply Protonated Peptides-Enhanced Sequence Coverage}

For several of the peptide ions examined, performing ETD/CID enhanced the already good coverage provided by ETD alone. For example, performing ETD on the triply protonated peptide KGAILKGAILR (MW = $1138.8 \mathrm{Da} ; \mathrm{m} / \mathrm{z} 380.6$ ) resulted in the formation and detection of 20 of the potential $22 \mathrm{c}$ - and z-type fragment ions for this peptide (Figure 2a). Performing ETD/CID increased this coverage level by forming 39 of a possible $44 \mathrm{~b}^{-}, \mathrm{y}^{-}, \mathrm{c}-$, , and $\mathrm{z}$-type fragment ions (Figure 2b). Similar sequence coverage was obtained whether the targeted auxiliary frequency was tuned to excite the unreacted precursor ion, $(\mathrm{M}+3 \mathrm{H})^{3+}$ at $\mathrm{m} / \mathrm{z}$ 380 , or the charge-reduced precursor ion, $(\mathrm{M}+2 \mathrm{H})^{2+}$ at $\mathrm{m} / \mathrm{z} 570$ (data not shown). Similar increases in overall sequence coverage were observed for many of the other peptides examined (Table 1), with a number of CIDderived fragment ions formed without much variation in the number of ETD-derived fragment ions observed. However, there were a few exceptions, including melittin and $\left[\mathrm{Glu}^{1}\right]$-fibrinopeptide, for two separate reasons (vide infra).

\section{ETD/CID of a Triply Protonated Phosphopeptide}

To examine the effectiveness of this method in the sequencing of peptides with post-translational modifications, ETD/CID was performed on the triply protonated monophosphopeptide from $\beta$-casein (MW = 2061.8 Da). While ETD alone (Figure 4a) provided only 16 of 32 possible c- and z-type fragment ions, ETD/CID

Figure 4. (a) ETD and (b) ETD/CID of a monophosphopeptide from $\beta$-casein tryptic digest. The ions selected for ETD and CID are indicated by the solid and dotted circles, respectively. (c) Mass spectrum showing the presence of both $\mathrm{b}$ - and $\mathrm{y}$-ions derived from the intact and dephosphorylated phosphopeptide ion, but only c-ions that are derived from the intact phosphopeptide ion. The $y$ - and z-type ions present are common to both phosphorylated and dephosphorylated analogues. 

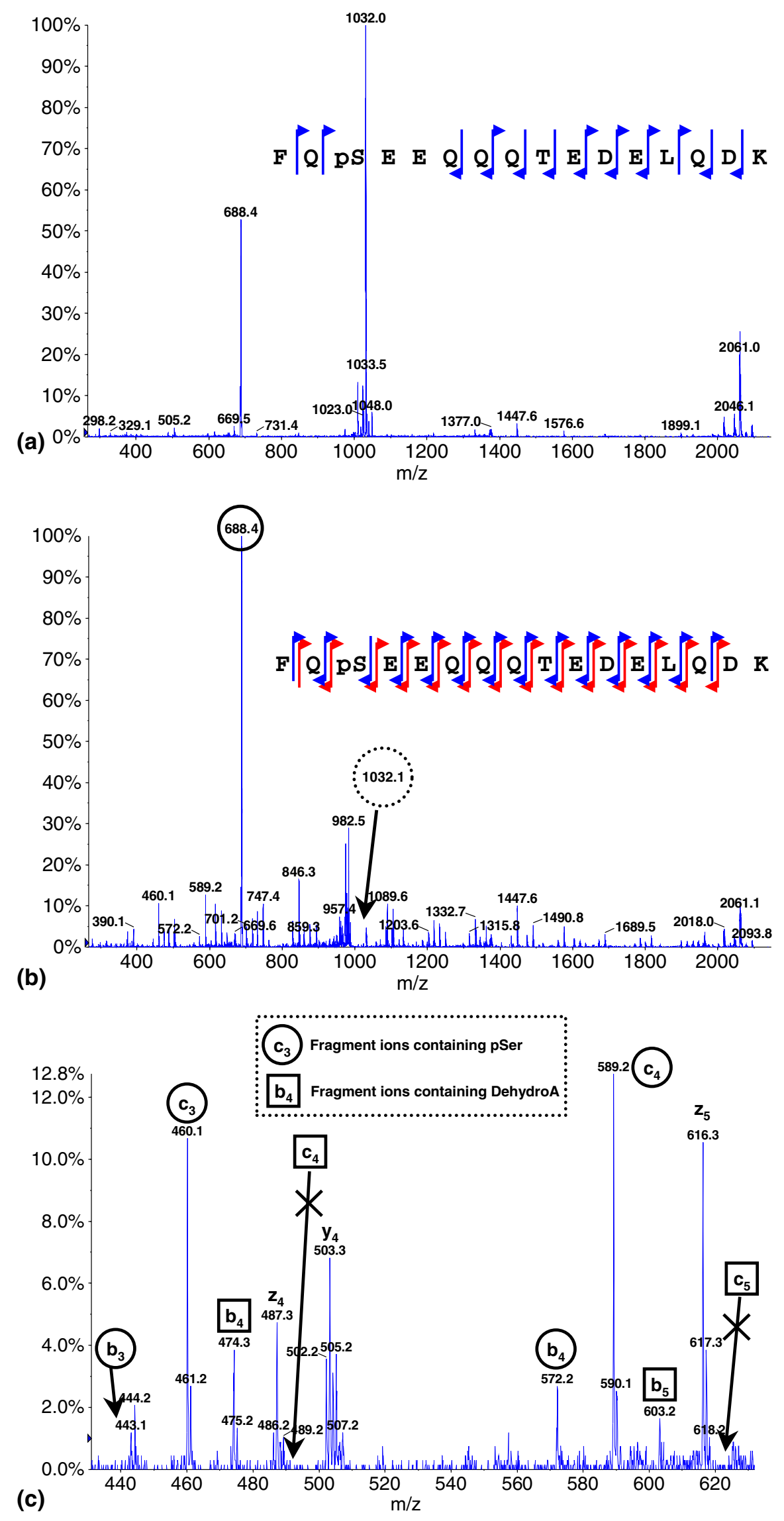
(excitation on the charge-reduced precursor ion of $\mathrm{m} / \mathrm{z}$ 1032) resulted in the formation of 60 of 64 possible fragment ions (Figure 4b). As expected, some b- and $y$-fragment ions were detected that are indicative of the dephosphorylated peptide cation, containing a dehydroalanine (DehydroA) residue in place of the original phosphoserine (pSer) (Figure 4c). This is typical of CID data for this class of molecule. Conversely, none of the observed c- and z-type fragment ions were derived from the dephosphorylated peptide ion (e.g., the $\mathrm{c}_{4}$ and $c_{5}$ fragments of the intact phosphopeptide ion are present, but not the dephosphorylated peptide ion). This indicates that the dephosphorylated peptide ion did not undergo any ETD reaction. However, the formation of $b$ - and y-type ions containing the intact phosphoserine group, along with the associated c- and z-type ions, are particularly intriguing, and will be the subject of further investigation.

\section{ETD/CID of Doubly Protonated Peptides-When ETD Needs Help}

For the doubly protonated peptides examined, application of the auxiliary activation (i.e., CID) on either the unreacted precursor ion or charge-reduced spe-
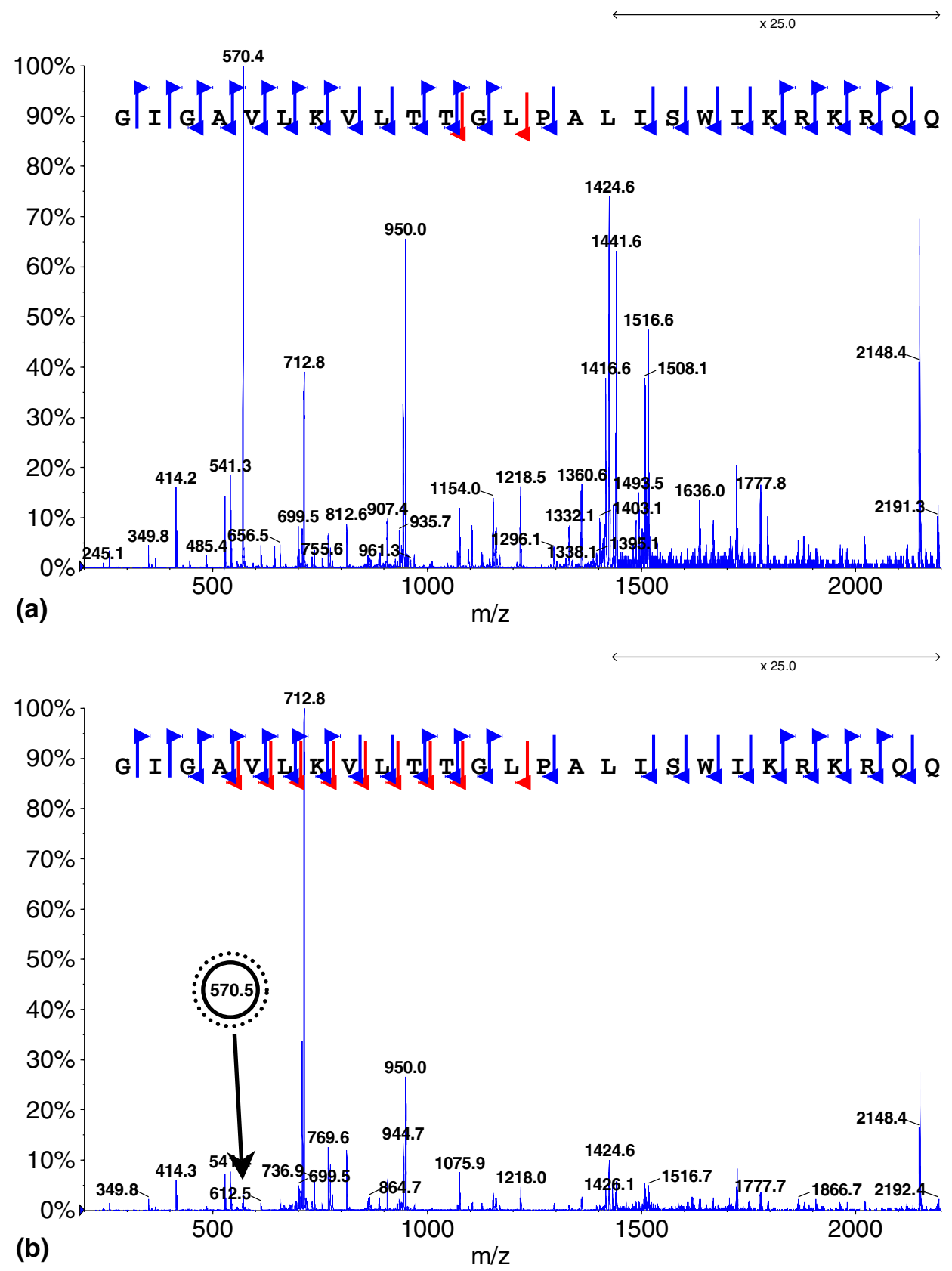

Figure 5. (a) ETD and (b) ETD/CID of the $(\mathrm{M}+5 \mathrm{H})^{5+}$ ion of melittin. The ions selected for ETD and CID are indicated by the solid and dotted circles, respectively. 
cies of the ETD event produces enhanced sequence coverage. Selection of the residual precursor ion yields b- and/or y-type ions (similar to those provided by CID-only MS/MS experiments). Alternatively, CID performed on the charge-reduced precursor ion can produce one of three effects: (1) augmented formation principally of c- and z-type ions, (2) formation principally of b- and y-type ions, or (3) augmented formation of all four types of peptide fragment ions.

The ETD/CID analysis of doubly protonated human $\left[\mathrm{Glu}^{1}\right]$-fibrinopeptide B provides an example of enhanced sequence coverage for a peptide resistant to fragmentation via ETD. When the $(\mathrm{M}+2 \mathrm{H})^{2+}$ ion $(\mathrm{m} / \mathrm{z}$ 785.8) was subjected to ETD-only, the generation of c- and z-type ions was quite inefficient with only three $z$-ions $\left(z_{12}\right.$ through $\left.z_{14}\right)$ observed (Figure 3a). In this case, activation of the residual precursor ion yielded different results than did activation of the charge-reduced precursor ion. ETD/CID performed on the charge-reduced species $(\mathrm{m} / \mathrm{z} 1570)$ provided seven $\mathrm{z}$-type ions $\left(\mathrm{z}_{7}\right.$ through $\left.\mathrm{z}_{13}\right)$, albeit of relatively low intensity (Figure $3 b$ ). Alternatively, ETD/CID performed on the residual precursor ion $(\mathrm{m} / \mathrm{z} 786)$ provided 24 of 28 predicted b- and y-type ions, in addition to the z-type ions, $\mathrm{z}_{12}$ through $\mathrm{z}_{14}$ (Figure $3 c$ ). While the ETD-derived ion population was not enhanced, the CID-derived product ions resulted in a rich fragment ion spectrum for peptide identification. Hence, in cases where ETD fragmentation is not favored due to amino acid sequence, charge state, or molecular weight (Angiotensin I also fit this scenario), the formation of CID-derived fragment ions produced in the ETD/CID event will provide similar coverage that would be expected from a CID-based MS/MS experiment. This rationale is similar to the "decision-tree" based methodology described by Swaney and coworkers [8] for analyzing small peptide ions of low charge state. However, ETD/CID relies upon the fundamental reactivity of the analyte cations to provide the best fragmentation, not computer logic or the associated two segregated scan types.

While CID-only MS/MS data could be acquired much faster than ETD/CID, there are potential applications for this technique. For example, ETD/CID could be used as an alternative to ETD-only experiments. This could benefit circumstances where ETD is selected based upon a priori logic, but fails to produce high-quality MS/MS spectra. The simultaneous CID event would provide another chance at sequencing such a peptide ion, while not having to increase the cycle time beyond an ETD-only experiment. In addition, other approaches require the acquisition of sequential ETD and CID mass spectra for some designated peptide ions-ETD/CID could acquire these data in a single mass spectrum.

\section{ETD/CID of a Larger, Highly Charged Peptide-When ETD Carries the Load}

For larger peptides and proteins with higher charge states $(z \geq 4)$, the data produced by the ETD/CID experiment again reflect the nature and reactivity of the ions involved. For example, the ETD-only mass spectrum of the $(\mathrm{M}+5 \mathrm{H})^{5+}$ ion of melittin $(\mathrm{m} / \mathrm{z} 570.4)$ reveals the formation of 37 of a possible $51 \mathrm{c}$ - and z-type fragment ions with a \% ETD of 56.8\% (Figure 5a). However, performing ETD/CID on this ion, with activation of any of the residual precursor ions, does not enhance the sequencing of this peptide to any great extent (Figure $5 b$ ). The number of ETD-derived fragment ions is not increased by the concomitant CID event, nor are the number of CID-derived fragment ions enhanced greatly. In this scenario, the multiply protonated peptide/protein has fragmented almost exclusively via ETD, not CID. However, given the high efficiency of ETD reactions involving highly charged peptide ions [2] based partly on the high rate constants for such ions [5], good sequence coverage of these cations is achieved. Further investigations will delve into the causes of this competition of fragment ion formation.

\section{The Influence of $\Delta \nu$ and $\Delta V$ on the Appearance of ETD/CID Mass Spectra}

The influence of altering the amplitude of the applied waveform during the ETD/CID experiment was examined using the triply protonated peptide KGAILKGAILR as an example. ETD-derived fragment ions (e.g., $z_{3}$ and $c_{4}$ ) appear in the mass spectrum without any amplitude on the auxiliary waveform, and are produced at approximately the same intensity regardless of the auxiliary waveform (Figure 6a). With increasing amplitude, CID-derived fragment ions begin to appear (e.g., $\left.\mathrm{y}_{7}\right)$, and become more intense with increasing voltage. However, at excessive voltages $\left(\geq 4.7 \mathrm{~V}_{p-p}\right.$ in this case), overall signal attenuation begins to set in due to the ions striking the rods after excessive excitation in a radial direction during the ETD/CID event.

The same selective activation is observed when the frequency of the applied auxiliary waveform is altered (Figure 6b). As the auxiliary waveform frequency comes into resonance with the secular frequency of the activated residual precursor ion (either the unreacted precursor ion or a charge-reduced precursor ion), the signals for the b- and y-type ions are influenced to a great extent. Their intensities exhibit maxima at the resonance frequency, falling off at other frequencies. Conversely, the intensities of the ETD-derived product ions are less affected. This can aid in the identification and verification of those fragments that are derived from a CID event as opposed to the ETD event. 

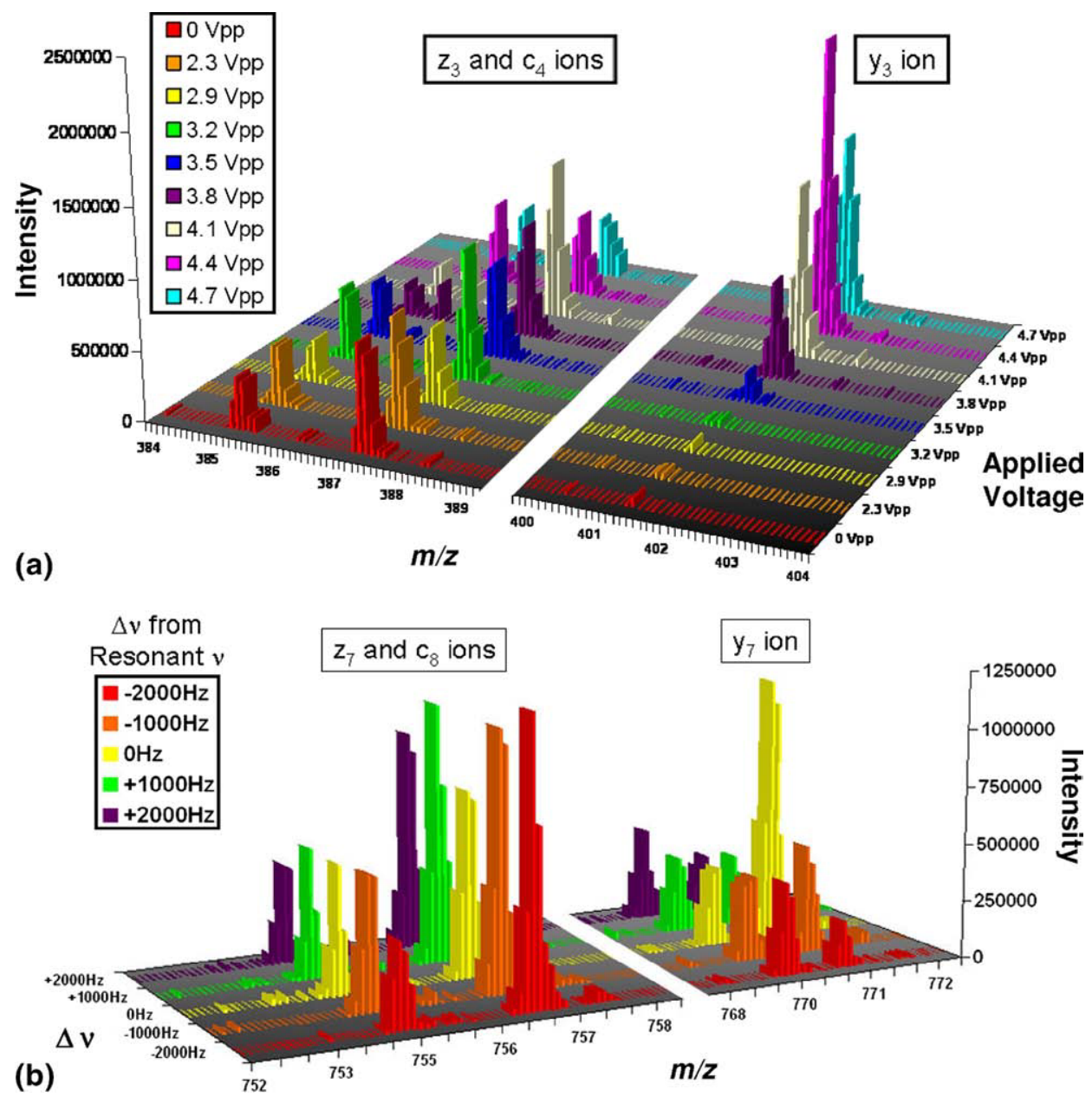

Figure 6. Plots showing the influence of altering the (a) amplitude and (b) frequency $(\Delta \nu)$ of the auxiliary voltage applied during the ETD/CID experiments. These data are from ETD/CID experiments on the triply protonated peptide KGAILKGAILR.

\section{Conclusions}

The simultaneous application of both ETD and CID shows promise as a tandem mass spectrometry method for sequencing peptides and proteins. ETD/CID appears to rely upon the fundamental reactivity of the analyte cations to provide the best fragmentation. Preliminary results demonstrate that ETD/CID can produce $b_{-}, y_{-}^{-}, c^{-}$, and $\mathrm{z}$-type ions in a single experiment that are displayed in a single mass spectrum. While some peptides, especially doubly protonated ones, do not fragment well by ETD, ETD/CID alleviates this problem by acting in one of three ways: (1) ETD fragment ions are enhanced by CID of chargereduced precursor ions, (2) both ETD and CIDderived fragments are produced, or (3) predominantly CID-derived fragments are produced with little or no ETD-derived fragment ions. Two interesting scenarios were presented that display the flexibility of the ETD/CID method. Smaller peptides that show little response to ETD are fragmented preferentially by CID during the ETD/CID experiment. Con- versely, larger peptides with higher charge states are fragmented primarily via ETD.

\section{Acknowledgments}

The authors acknowledge Professor Scott McLuckey of Purdue University for insightful discussions, and Professor Ivan Chu of The University of Hong Kong for helping to stimulate this work. They thank Dr. Frank A. Londry for providing hardware support and the SxStability software, Dr. Brad Schneider for advice on the DuoSpray ion source, Dr. Min Yang for assistance with MS Expo software, and Dr. Stephen Tate and Dr. David Cox for stimulating discussions and invaluable input.

\section{References}

1. Zubarev, R. A.; Kelleher, N. L.; McLafferty, F. W. Electron capture dissociation of multiply charged protein cations. A nonergodic process. J. Am. Chem. Soc. 1998, 120, 3265-3266.

2. Syka, J. E. P.; Coon, J .J.; Schroeder, M. J.; Shabanowitz, J.; Hunt, D. F. Peptide and protein sequence analysis by electron transfer dissociation mass spectrometry. Proc. Nat. Acad. Sci. U.S.A. 2004, 101, 9528-9533.

3. Pitteri, S. J.; Chrisman, P.A.; Hogan, J. M.; McLuckey, S. A. Electron transfer ion/ion reactions in a three-dimensional quadrupole ion trap: Reactions of doubly and triply protonated peptides with $\mathrm{SO}_{2}{ }^{\bullet-}$. Anal. Chem. 2005, 77, 1831-1839. 
4. Pitteri, S. J.; Chrisman, P. A.; McLuckey, S. A. Electron transfer ion/ion reactions of doubly protonated peptides: Effect of elevated bath gas temperature. Anal. Chem. 2005, 77, 5662-5669.

5. McLuckey, S. A.; Stephenson, J. L. Jr. Ion/ion chemistry of high-mass multiply charged ions. Mass Spectrom. Rev. 1998, 17, 369-407.

6. McAlister, G. C.; Berggren, W. T.; Griep-Raming, J.; Horning, S.; Makarov, A.; Phanstiel, D.; Stafford, G.; Swaney, D. L.; Syka, J. E. P.; Zabrouskov, V.; Coon, J. J. A proteomics grade electron transfer dissociation-enabled hybrid linear ion trap-Orbitrap mass spectrometer. J. Proteome Res. 2008, 7, 3127-3136.

7. Chi, A.; Huttenhower, C.; Geer, L.Y.; Coon, J. J.; Syka, J. E. P.; Bai, D. L.; Shabanowitz, J.; Burke, D. J.; Troyanskaya, O. G.; Hunt, D. F. Analysis of phosphorylation sites on proteins from Saccharomyces cerevisiae by electron transfer dissociation (ETD) mass spectrometry. Proc. Natl. Acad. Sci. U.S.A. 2007, 104, 2193-2198.

8. Swaney, D. L.; McAlister, G. C.; Coon, J. J. Decision tree-driven tandem mass spectrometry for shotgun proteomics. Nature Methods 2008, 5, 959-964.

9. Xia, Y.; Gunawardena, H. P.; Erickson, D. E.; McLuckey, S. A. Effects of cation charge-site identity and position on electron-transfer dissociation of polypeptide cations. J. Am. Chem. Soc. 2007, 129, 12232-12243.

10. Chrisman, P. A.; Pitteri, S. J.; McLuckey, S. A. Parallel ion parking: Improving conversion of parents to first-generation products in electron transfer dissociation. Anal. Chem. 2005, 77, 3411-3414.

11. Han, H.; Xia, Y.; McLuckey, S. A. Beam-type collisional activation of polypeptide cations that survive ion/ion electron transfer. Rapid Commun. Mass Spectrom. 2007, 21, 1567-1573.

12. Han, H.; Xia, Y.; McLuckey, S. A. Ion trap collisional activation of $\mathrm{c}$ and $\mathrm{z}^{\bullet}$ Ions formed via gas-phase ion/ion electron-transfer dissociation. J. Proteome Res. 2007, 6, 3062-3069.

13. Swaney, D. L.; McAlister, G. C.; Wirtala, M.; Schwartz, J. C.; Syka, J. E. P.; Coon, J. J. Supplemental activation method for high-efficiency electron-transfer dissociation of doubly protonated peptide precursors. Anal. Chem. 2007, 79, 477-485.

14. Xia, Y.; Han, H.; McLuckey, S. A. Activation of intact electron-transfer products of polypeptides and proteins in cation transmission mode ion/ion reactions. Anal. Chem. 2008, 80, 1111-1117.

15. Coon, J. J.; Syka, J. E. P.; Schwartz, J. C.; Shabanowitz, J.; Hunt, D. F. Anion dependence in the partitioning between proton and electron transfer in ion/ion reactions. Int. J. Mass Spectrom. 2004, 236, 33-42.

16. Gunawardena, H. P.; He, M.; Chrisman, P. A.; Pitteri, S. J.; Hogan, J. M.; Hodges, B. D. M.; McLuckey, S. A. Electron transfer versus proton transfer in gas-phase ion/ion reactions of polyprotonated peptides. J. Am. Chem. Soc. 2005, 127, 12627-12639.

17. Horn, D. M.; Zubarev, R. A.; McLafferty, F. W. Automated de novo sequencing of proteins by tandem high-resolution mass spectrometry. Proc. Nat. Acad. Sci. U.S.A. 2000, 97, 10313-10317.

18. Nielsen, M. L.; Savitski, M. M.; Zubarev, R. A. Improving protein identification using complementary fragmentation techniques in Fourier transform mass spectrometry. Mol. Cell. Proteom. 2005, 4, 835-845.

19. Zubarev, R. A.; Zubarev, A. R.; Savitski, M. M. Electron capture/ transfer versus collisionally activated/induced dissociations: Solo or duet? J. Am. Soc. Mass Spectrom. 2008, 19, 753-761.

20. Hogan, J. M.; Pitteri, S. J.; Chrisman, P. A.; McLuckey, S. A. Complementary structural information from a tryptic $N$-linked glycopeptides via electron transfer ion/ion reactions and collision-induced dissociation. J. Proteome Res. 2005, 4, 628-632.

21. Han, H.; Xia, Y.; Yang, M.; McLuckey, S. A. Rapidly alternating transmission mode electron-transfer dissociation and collisional activation for the characterization of polypeptide ions. Anal. Chem. 2008, 80, 3492-3497.

22. Gorshkov, M. V.; Masselon, C. D.; Nikolaev, E. N.; Udseth, H. R.; Paša-Tolić, L.; Smith, R. D. Considerations for electron capture dissoci- ation efficiency in FTICR mass spectrometry. Int. J. Mass Spectrom. 2004, 234, 131-136.

23. Horn, D. M.; Ge, Y.; McLafferty, F. W. Activated ion electron capture dissociation for mass spectral sequencing of larger $(42 \mathrm{kDa})$ proteins. Anal. Chem. 2000, 72, 4778-4784.

24. Tsybin, Y. O.; Witt, M.; Baykut, G.; Kjeldsen, F.; Håkansson, P. Combined infrared multiphoton dissociation and electron capture dissociation with a hollow electron beam in Fourier transform ion cyclotron resonance mass spectrometry. Rapid Commun. Mass Spectrom. 2003, 17, $1759-1768$.

25. Mihalca, R.; van der Burgt, Y. E. M.; McDonnell, L. A.; Duursma, M Cerjak, I.; Heck, A. J. R.; Heeren, R. M. A. Combined infrared multiphoton dissociation and electron-capture dissociation using co-linear and overlapping beams in Fourier transform ion cyclotron resonance mass spectrometry. Rapid Commun. Mass Spectrom. 2006, 20, 1838-1844.

26. Liu, J.; Gunawardena, H. P.; Huang, T.-Y.; McLuckey, S. A. Chargedependent dissociation of insulin cations via ion/ion electron transfer. Int. J. Mass Spectrom. 2008, 276, 160-170.

27. Håkansson, K.; Chalmers, M. J.; Quinn, J. P.; McFarland, M. A. Hendrickson, C. L.; Marshall, A. G. Combined electron capture and infrared multiphoton dissociation for multistage MS/MS in a Fourier transform ion cyclotron resonance mass spectrometer. Anal. Chem. 2003, $75,3256-3262$.

28. Good, D. M.; Wirtala, M.; McAlister, G. C.; Coon, J. J. Performance characteristics of electron transfer dissociation mass spectrometry. Mol. Cell. Proteom. 2007, 6, 1942-1951.

29. Kaplan, D. A.; Hartmer, R.; Speir, J. P.; Stoermer, C.; Gumerov, D.; Easterling, M.L.; Brekenfeld, A.; Kim, T.; Laukien, F.; Park, M. A. Electron transfer dissociation in the hexapole collision cell of a hybrid quadrupole-hexapole Fourier transform ion cyclotron resonance mass spectrometer. Rapid Commun. Mass Spectrom. 2008, 22, 271-278.

30. McAlister, G. C.; Phanstiel, D.; Good, D. M.; Berggren, W. T.; Coon, J. J. Implementation of electron-transfer dissociation on a hybrid linear ion trap-Orbitrap mass spectrometer. Anal. Chem. 2007, 79, 3525-3534.

31. Williams, D. K. Jr.; McAlister, G. C.; Good, D. M.; Coon, J. J.; Muddiman, D. C. Dual electrospray ion source for electron-transfer dissociation on a hybrid linear ion trap-Orbitrap mass spectrometer. Anal. Chem. 2007, 79, 7916-7919.

32. Brown, J.; Campuzano, I.; Pringle, S.; Chapman, R. Electron transfer dissociation within a rf traveling wave ion guide collision cell of a QTOF. Proceedings of the 56th Annual Meeting of the American Society for Mass Spectrometry Conference on Mass Spectrometry and Allied Topics; Denver, CO, June 2008.

33. Stanley, S. M. R.; Foo, H. C. Screening for basic drugs in equine urine using direct-injection differential-gradient LC-LC coupled to hybrid tandem MS/MS. J. Chromatogr. B 2006, 836, 1-14.

34. Hager, J. W. Off-resonance excitation in a linear ion trap. J. Am. Soc. Mass Spectrom. 2009, 20, 443-450.

35. Liang, X.; Xia, Y.; McLuckey, S. A. Alternately pulsed nanoelectrospray ionization/atmospheric pressure chemical ionization for ion/ion reactions in an electrodynamic ion trap. Anal. Chem. 2006, 78, 3208-3212.

36. Thomson, B. A.; Jolliffe, C. L. Spectrometer with axial field. U.S. Patent 5,847,386, December 8, 1998.

37. Loboda, A.; Krutchinsky, A.; Loboda, O.; McNabb, J.; Spicer, V.; Ens, W.; Standing, K. G. Novel LINAC II electrode geometry for creating an axial field in a multipole ion guide. Eur. J. Mass Spectrom. 2000, 6, 531-536.

38. Londry, F. A.; Hager, J. W. Mass selective axial ion ejection from a linear quadrupole ion trap. J. Am. Soc. Mass Spectrom. 2003, 14, 1130-1147.

39. Stephenson, J. L. Jr.; McLuckey, S. A. Anion effects on storage and resonance ejection of high mass-to-charge cations in quadrupole ion trap mass spectrometry. Anal. Chem. 1997, 69, 3760-3766. 